\title{
Egouttement en jeunes pessières et intensité de la première éclaircie
}

\author{
F. FRANÇOIS, P. MERTENS, P. ANDRÉ et F. DEVILLEZ * \\ Laboratoire forestier de l'Université catholique de Louvain \\ Faculté des Sciences agronomiques \\ 2, place Croix-du-Sud, B.P. 4, B 1348 Louvain-la-Neuve, Belgique \\ * Laboratoire d'Ecologie végétale de l'Université catholique de Louvain \\ Faculté des Sciences \\ 4, place Croix-du-Sud, B 1348 Louvain-la-Neuve, Belgique
}

\section{Résumé}

L'intensité de la première éclaircie en jeunes pessières conditionne directement la production courante du peuplement en modifiant notamment l'environnement de croissance des arbres.

Une des composantes du bilan hydrique - l'égouttement - a été mesurće au cours de la période de végétation sous le couvert de 2 peuplements de densités différentes (16,5 et $\left.24 \mathrm{~m}^{2} / \mathrm{ha}\right)$ traités par éclaircie sélective.

Les principaux résultats font état d'une augmentation de 8,4 p. 100 du taux d'égouttement dans la parcelle fortement éclaircic, où, par ailleurs, il a été constaté que le seuil d'égouttement moyen diminuait de 27 p. 100.

Le maintien ou l'amélioration du taux de croissance des épicéas, soumis à de fortes éclaircies, pourrait done s'expliquer en partic par l'apport d'une quantité d'eau supplémentaire, en période de sécheresse relative.

En pessières équiennes de 20 à 30 ans, de récentes expériences (ANDRÉ, 1976 ; ANDRÉ \& BLEROT, 1978) démontrent qu'une première éclaircie, dont l'intensité dépasse sensiblement les normes généralement retenues dans le traitement de l'épicéa, favorise la croissance des arbres et des peuplements et cela, malgré une perturbation complète de l'état de massif. Cette augmentation de croissance résulte de la double action de l'éclaircie sur le peuplement : diminution de la compétition interindividuelle d'une part, et modifications quantitatives et qualitatives du milieu de croissance d'autre part.

Dans cette optique, nous avons abordé, en relation directe avec le traitement sylvicole, les variations de certaines composantes mésologiques (quantités de précipitations reçues sous le couvert, rayonnement perçu au niveau du sol et température de la couche superficielle du substrat) en vue de discuter leur effet sur la croissance et d'interpréter le rôle de l'éclaircie. Aussenac et al. (1982) ont publié une étude 
relative à l'influence d'une éclaircie systématique sur le bilan hydrique d'un peuplement de douglas âgés de 19 ans. Plus précisément, cette note est consacrée aux mesures des quantités de précipitations atteignant le sol, pendant la période de croissance, sous le couvert de 2 peuplements d'épicéas traités selon 2 intensités d'éclaircies sélcctives extrêmes.

\section{Matériel et méthode}

Deux parcelles de densités différentes ont été choisies dans le dispositif mis en œuvre par le Laboratoire forestier de l'Université catholique de Louvain, et situé dans le bois de Lauzelle à une altitude de $110 \mathrm{~m}$ (province de Brabant, Belgique), sur sol limoneux du type Aba (classification belge). Les principales caractéristiques de ces parcelles sont reprises au tableau 1. A 27 ans, la hauteur dominante des épicéas était de 18 mètres. Chaque parcelle a une superficie d'environ 8 ares. Plantés en 1951 à 1,5 m d'écartement, ces épicéas ont été éclaircis pour la première fois en mars 1977.

\section{TABleau 1}

Caractéristiques des peuplements de la parcelle A (intensité d'éclaircie normale) et $B$ (forte intensité d'éclaircie).

Properties of stands : parcel A (light intensity of thinning) and parcel $B$ (heal'y intensity of thinning).

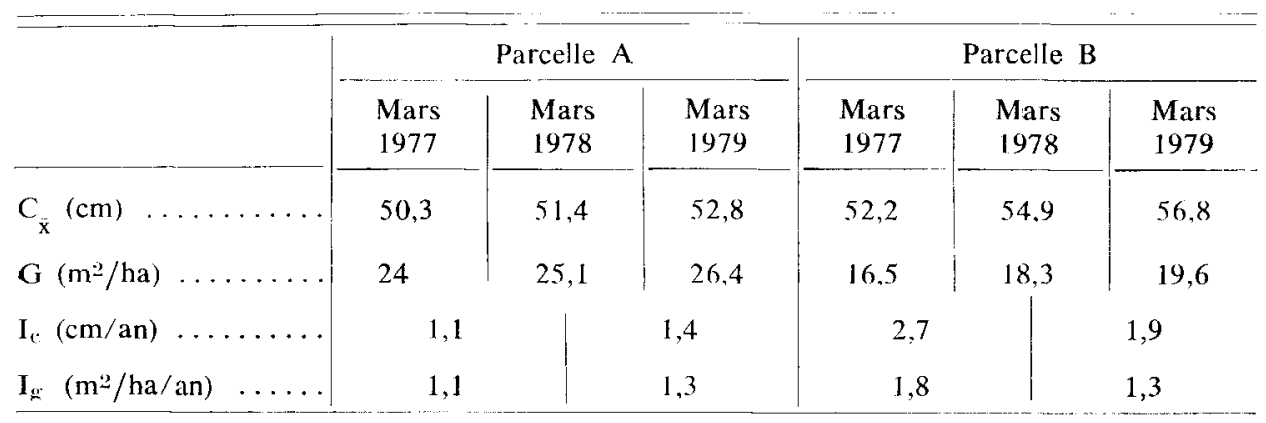

Avec - With:

C : Circonférence moyenne des arbres. Mean girth of trees.

G : Surface terrière. Basal area.

$\mathrm{I}_{\mathbf{c}} \quad$ : Accroissement courant en circonférence. Current increment of girth.

$I_{g} \quad$ : Accroissement courant en surface terrière. Curent increment of basal area. 
Les mesures ont porté uniquement sur la fraction des précipitations atteignant le sol, communément appelée l'égouttement, à l'exclusion de l'eau qui s'écoule le long des troncs (fig. 1). Pendant la période estivale, on peut considérer que cette fraction des précipitations est négligeable par rapport à la quantité des précipitations incidentes. Aussenac (1968) cite des pourcentages d'écoulement qui varient cntre 0,1 et 1,3 p. 100 pour les mois de juin, juillet et août 1965 et 1966.

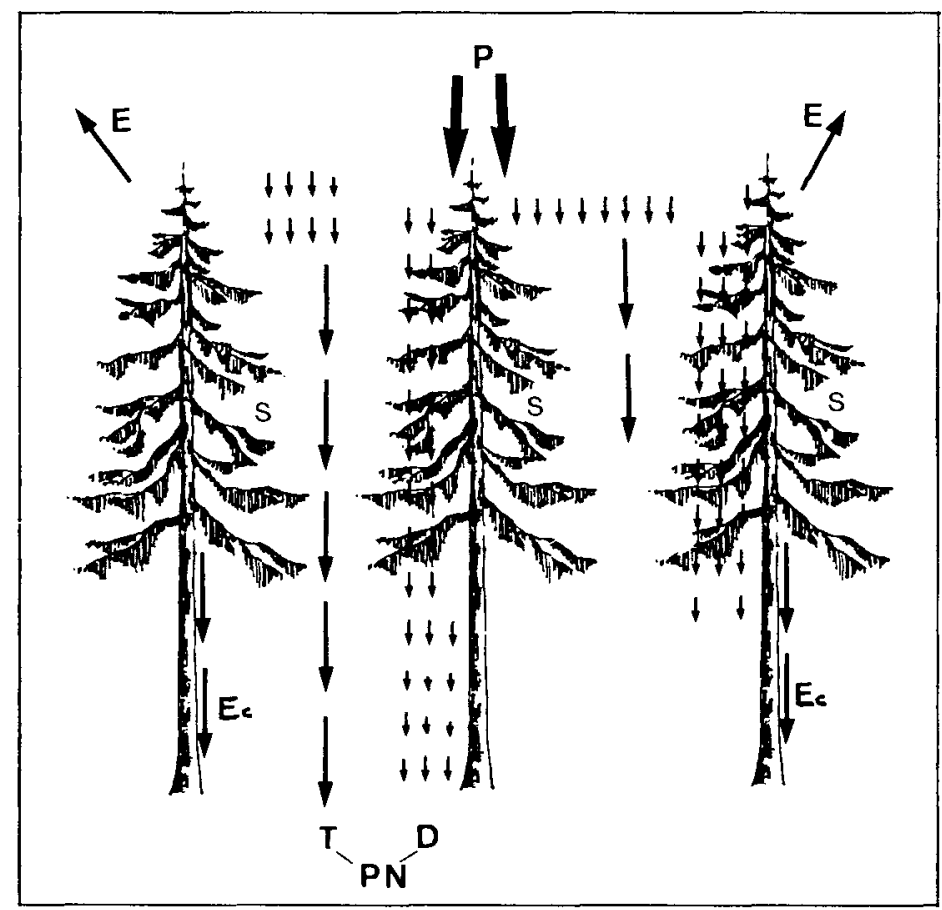

FIG. 1

Décomposition du budget des précipitations dans un écosystème forestier (inspiré de Leonard, 1961).

Decomposition of the total rainfalls in a forest ecosystem (from LEONARD, 1961).

Avec - With :

$\mathrm{P}$ : Précipitations incidentes au peuplement. Incident rainfall to the ecosystem.

$\mathrm{T}$ : Précipitations atteignant directement le sol. Rainfall reaching directly the ground.

D : Précipitations arrêtées par les cimes et qui tombent au sol par la suite. Rainfall stopped by the canopy layer and reaching the ground after.

S : Eau absorbée par les parties aériennes. Water absorbated by the aerial parts of the trees.

Ec : Précipitations qui s'écoulent le long du tronc. Rainfall flowing along the boles.

E : Quantités d'eau perdues par évaporation des surfaces végétales. Part of the water evaporated from the vegetation surfaces.

PN : Egouttement. Drip : $T+D$. 
Les précipitations ont été recueillies par des pluviomètres dont la surface totale de réception était identique dans chacune des parcelles, mais dont l'aire de répartition variait en fonction de l'hétérogénéité du couvert : les 20 pluviomètres disposés dans chaque parcelle étaient répartis sur une surface de $40 \mathrm{~m}^{2}$ dans la parcelle $B$ et de $25 \mathrm{~m}^{2}$ dans la parcelle $A$. Leur disposition est représentée de manière plus précise aux figures 3 et 4 , où la surface de projection des cimes est calculée sur base d'un rapport $\mathrm{D} / \mathrm{d}$ égal à 16 . L'égouttement a été mesuré une fois par semaine pendant 2 périodes de végétation successives (1977 et 1978), correspondant chacune à la phase de croissance secondaire des arbres. Le choix de cette période de mesure découle directement de l'objectif de cette étude, qui vise à interpréter les variations de l'accroissement en circonférence en fonction du traitement appliqué. Les données du poste climatologique ont été fournies par la station météorologique de 1'Université de Louvain, située à quelque $500 \mathrm{~m}$ du dispositif, et ont été assimilées aux quantités de précipitations atteignant le sommet du peuplement.

\section{Résultats et discussion}

Le tableau 2 et la figure 2 reprennent les valeurs de l'égouttement et des taux d'égouttement observés dans les parcelles $\mathrm{A}$ et $\mathrm{B}$, et relatives aux 2 périodes d'observation. Malgré des différences très nettes entre les rythmes de précipitations estivales des 2 années, les taux d'égouttement $\left(T_{\mathrm{F}}\right)$ restent constants et sont en moyenne supérieurs de 8,4 p. 100 dans la parcelle fortement éclaircie. Comparées aux valeurs proposées par la littérature (tabl. 4), les valeurs de l'égouttement obtenues dans notre expérience sont très faibles. Cet écart provient des caractéristiques inhérentes à la

\section{TABLEAU 2}

Egouttements ( $P N$ exprimés en $m m$ ) et taux d'égouttement (TE exprimé en p. 100) calculés pour chacune des parcelles faiblement $(A)$ et fortement $(B)$ éclaircies, et enregistrés au poste climatologique.

en fonction de la période d'observation et en moyenne pour ces deux périodes.

Drip (PN in $\mathrm{mm})$ and drip rate (TE in $P$. 100) calculated for each stand : light-thinned $(A)$ and heavy-thinned $(B)$, and recorded at the climatology station during each observation's season, and mean values for these two seasons.

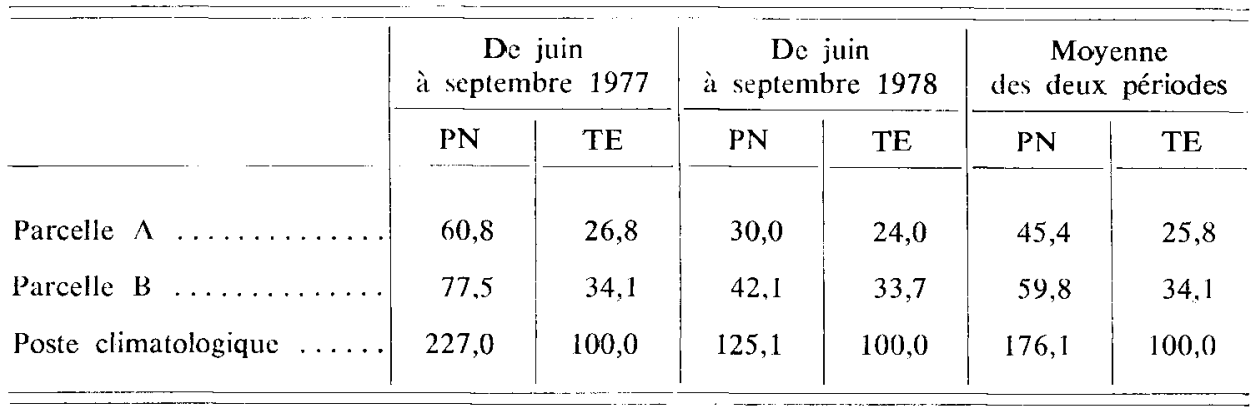


période de mesures - alternance de courtes ondées et de fortes chaleurs -. De telles conditions favorisent l'évaporation d'une partie de l'eau receuillie par les pluviomètres, le phénomène étant d'autant plus accusé, - et c'est le cas ici — que la périodicité des observations est assez longue. A titre de comparaison, Aussenac (1968) cite des valeurs d'interception nette de 59,6 et 51,5 p. 100 pour les mois d'août 1965 et juillet 1966, pour un peuplement d'épicéas de 30 ans. Mais ses observations ont été effectuées 4 ans après le passage en éclaircie de telle sorte que le couvert s'y était déjà refermé, ce qui n'est pas le cas dans notre expérience.

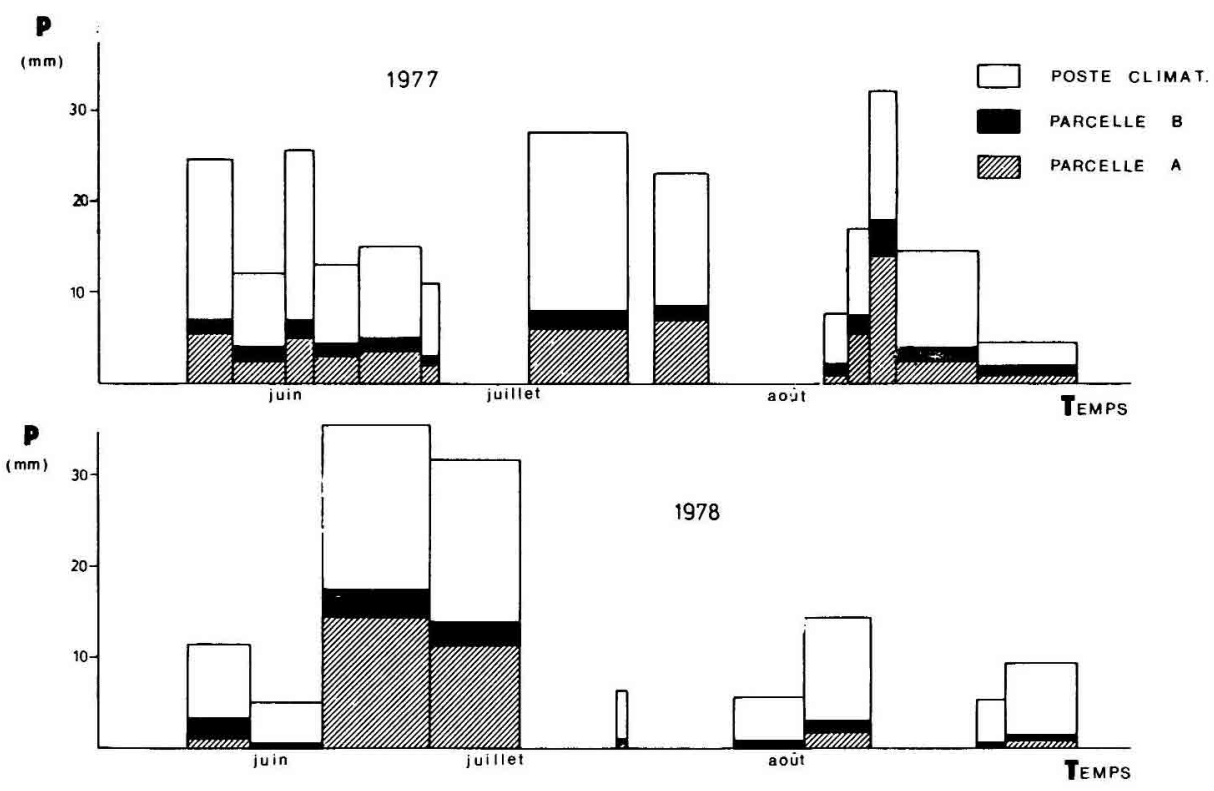

FIG. 2

Histogramme des quantités de précipitations.

Histogramm of rainfalls.

TABLEAU 3

Paramètres des régressions entre les précipitations incidentes au peuplement (P) et l'égouttement $(P N)$ en fonction de la période d'observation et de lintensité de léclaircie (A : parcelle faiblement éclaircie, $B$ : parcelle fortement éclaircie), $r$ représentant le coefficient de corrélation des équations.

Regression equations between the incident rainfalls to the ecosystem $(P)$ and the drip (PN) during each observation's season and for the two thinning treatments

( $A$ : light-thinned stand, $B$ : heavy-thinned stand), $r$ is the correlation coefficient of equations.

\begin{tabular}{c|c|c|c}
\hline Période & Parcelle & Equations de régression & $\mathbf{r}$ \\
\cline { 2 - 4 } 1977 & A & PN $=0,42 \mathrm{P}-2,87$ & $0,87^{* *}$ \\
& B & PN $=0,47 \mathrm{P}-2,27$ & $0,84^{* *}$ \\
1978 & A & PN $=0,45 \mathrm{P}-2,59$ & $0,91^{* *}$ \\
& B & PN=0,52 P $-2,36$ & $0,96^{* *}$ \\
\hline
\end{tabular}




\section{TABleau 4}

Données extraites de la littérature et relatives à la décomposition du budget des précipitations en peuplement résineux.

Results of the bibliography about the decomposition of the total rainfalls in softwood stands.

\begin{tabular}{|c|c|c|c|c|c|c|c|c|c|}
\hline & \multicolumn{4}{|c|}{$\begin{array}{l}\text { Caractéristiques } \\
\text { des peup'ements }\end{array}$} & \multirow{2}{*}{$T_{l i}$} & \multirow{2}{*}{$\mathrm{TI}$} & \multirow{2}{*}{ TE } & \multirow{2}{*}{\multicolumn{2}{|c|}{$\mathrm{SE}$}} \\
\hline & Age & $\mathrm{Nb} / \mathrm{ha}$ & $G$ & $\mathrm{H}_{\dot{\mathrm{x}}}$ & & & & & \\
\hline AUSSENAC (1968) & 30 & 2160 & - & 12.5 & 1,3 & 34,0 & 63,7 & 3,1 & $+0,8$ \\
\hline BuLTot et al. (1972) & 30 & 1500 & - & 16 & - & 20,5 & - & 5,2 & +0.5 \\
\hline WEIHE- (1968) & 59 & - & - & 27 & 一 & - & - & 6,5 & \\
\hline REYNOLDS et al. (1967) & 25 & - & 26 & - & 一 & - & - & 3,5 & $+0,2$ \\
\hline NOIRFALISE (1959) & - & - & - & 22 & - & 38 & - & & - \\
\hline BRECHTEL (1969) & 54 & 400 & 35 & 15,2 & 1,0 & 40 & 59,0 & & — \\
\hline DeLFs $(1967) \ldots \ldots$ & 19 & - & 一 & 一 & - & 71,0 & 一 & 4,49 & +0.51 \\
\hline AUSSENAC et al. (1982) & & & & & & & & & \\
\hline - Eclaircie ..... & 19 & 1447 & 19,85 & 11,5 & 7,9 & 30.3 & 61,8 & & - \\
\hline 一 Témoin .... & 19 & 2932 & 39,27 & 11,5 & 10,8 & 43,4 & 45,8 & & - \\
\hline
\end{tabular}

Avec - With :

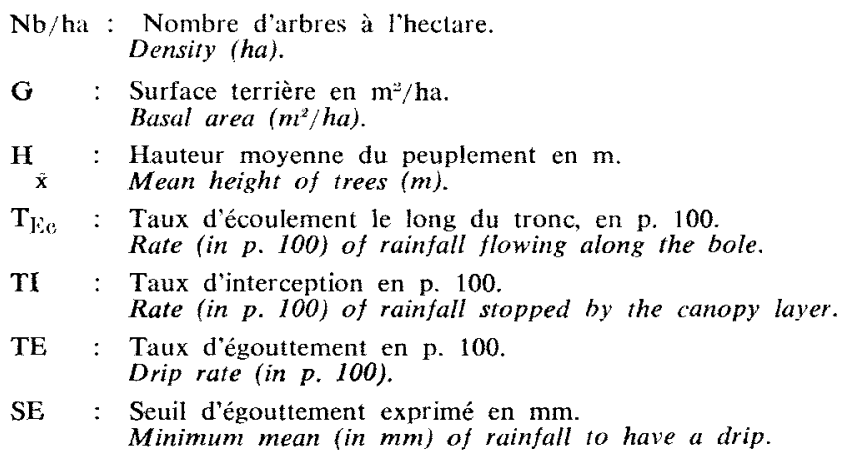

Les résultats inscrits au tableau 2 sont donc logiques, si l'on sait que, d'une part, les densités de nos parcelles A et B sont faibles et que d'autre part les mestres ne sont effectuées que pendant la période de croissance secondaire des arbres.

D'après Mrtscherlich \& Moll (1970), il existe pour chaque type de couvert forestier, une relation linéaire entre les précipitations atteignant le sommet du peuplement $(P)$ et l'égouttement $(P N): P N=a P+b(1)$ où $a$ et $b$ sont des constantes dépendant du type de précipitation et des caractéristiques du peuplement, et où $P$ et PN sont exprimés en $\mathrm{mm}$. Cette relation a été appliquée à nos résultats et les paramètres de l'équation (1) sont repris at tableau 2 pour chacune des parcelles et en fonction de la période de mesures. Notons de suite que les coefficients de 
corrélation sont hautement significatifs, ce qui confirme la relation existant entre l'égouttement et les précipitations incidentes au peuplement. Ces régressions nous permettent également de calculer les seuils d'égouttement $\left(S_{\mathrm{E}}\right)$ exprimés en $\mathrm{mm}$; ils représentent les quantités de précipitations nécessaires à la saturation du feuillage avant le début de l'égouttement. En effet, d'après Kalma et al. (1968), on a :

$$
\mathrm{SE}=-\frac{\mathrm{b}}{\mathrm{a}} \quad \text { pour } \quad \mathrm{PN}=0
$$

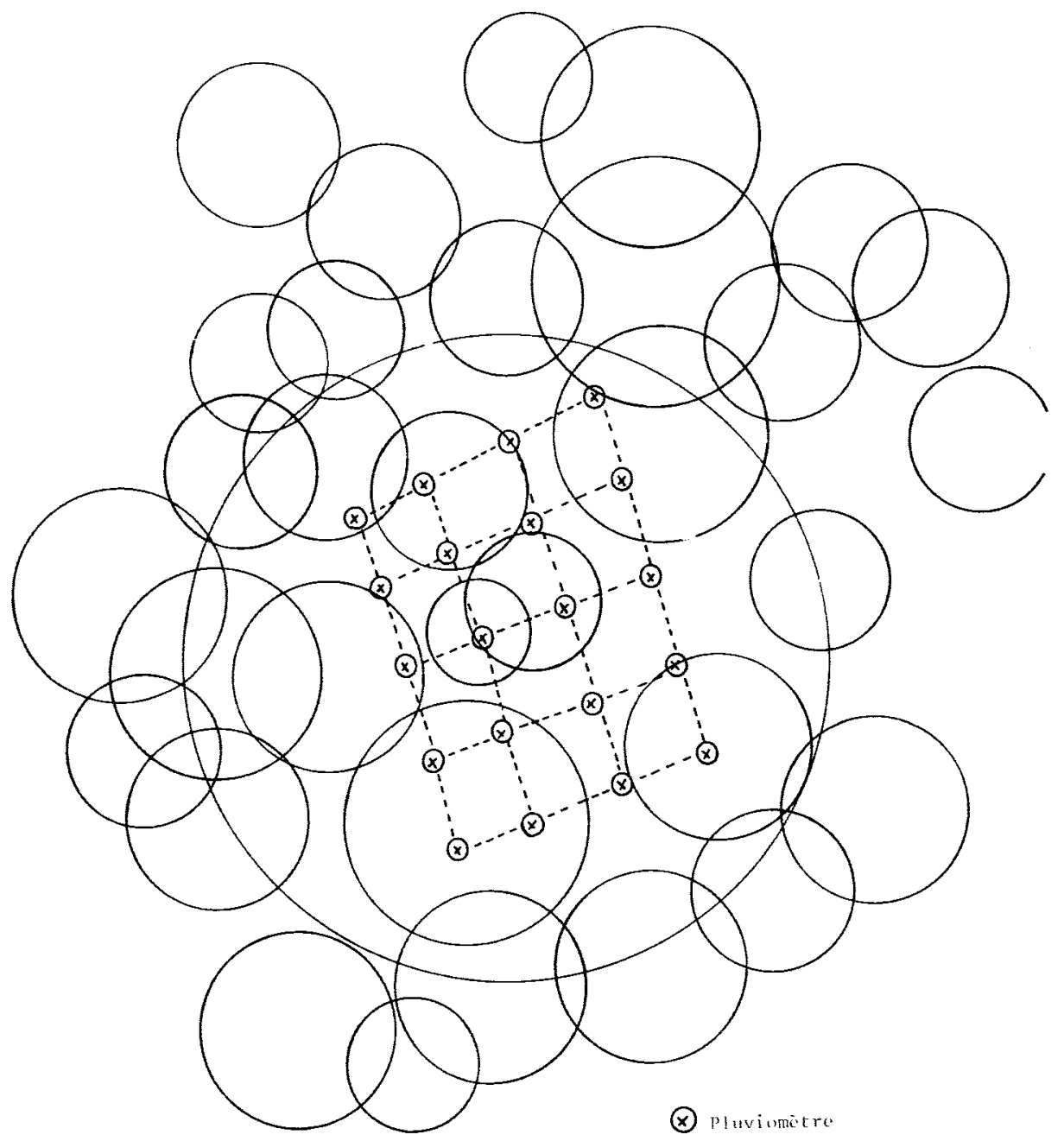

Fig. 3

Représentation schématique de la disposition des pluviomètres sous le couvert des arbres de la parcelle $A$.

Scheme of the lay out of the rain gauges under the trees layer of stand $A$. 


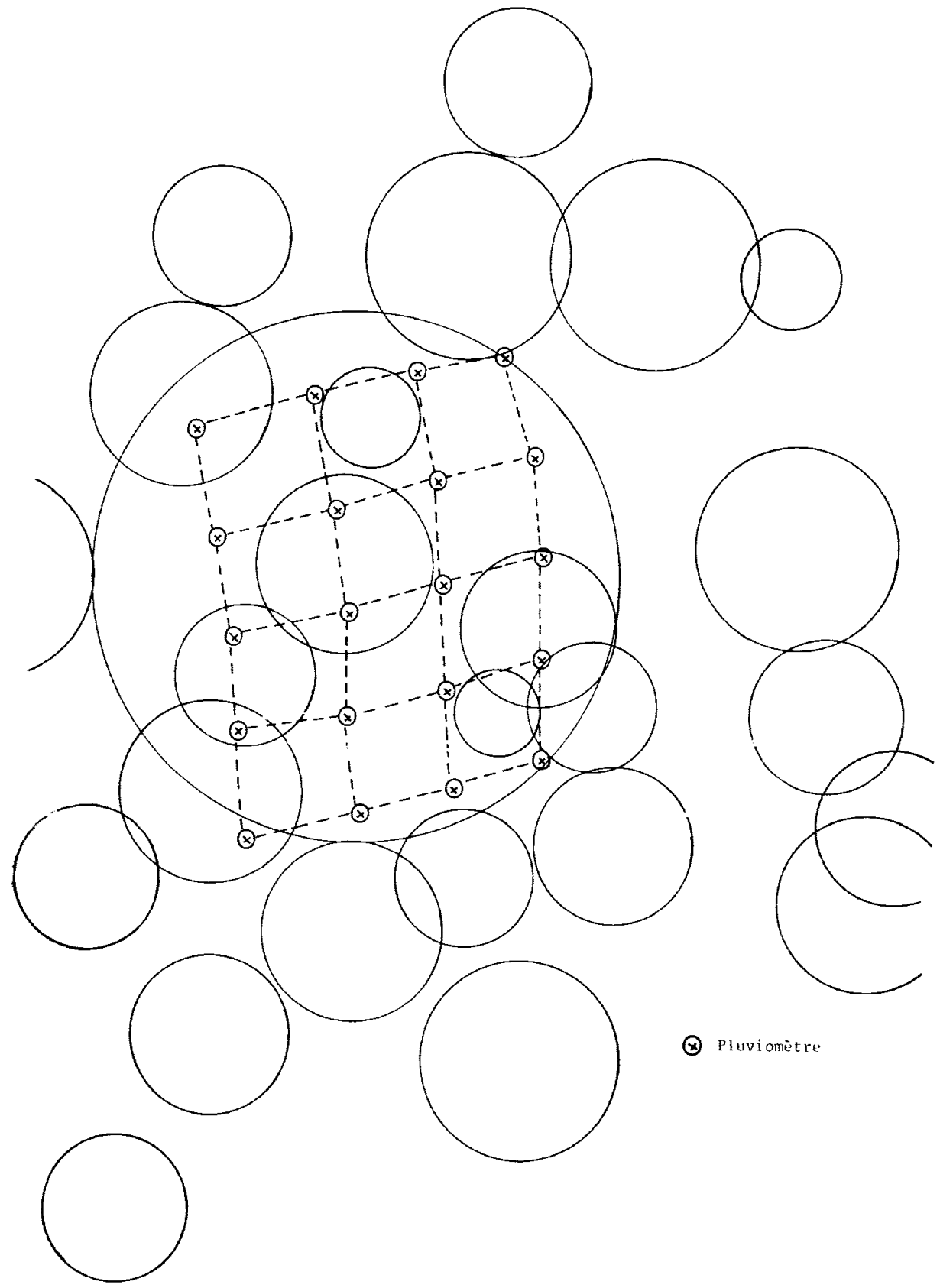

Fig. 4

Représentation schématique de la disposition des pluviomètres sous le couvert des arbres de la parcelle $B$.

Scheme of the lay out of the rain gauges under the trees layer of stand $B$. 
Appliquée à nos parcelles, cette relation donne les résultats suivants :

\begin{tabular}{|c|c|c|}
\hline & Parcelle A & Parcelle B \\
\hline 1977 & $6,79 \pm 0,82$ & $4,87 \pm 0,60$ \\
\hline 1978 & $5,81 \pm 0,70$ & $4,49 \pm 0,51$ \\
\hline
\end{tabular}

Ces valeurs s'inscrivent dans l'intervalle des seuils généralement admis et qui s'étend entre 3 et $7 \mathrm{~mm}$ (tabl. 4). Il importe d'insister sur la différence qui se manifeste ici entre les parcelles de densités extrêmes. On constate en effet que, pour des pluies dont l'intensité a été comprise entre 4,6 et $6,3 \mathrm{~mm}$ - scuils d'égouttement moyens calculés sur les 2 périodes - l'égouttement n'a eu lieu que dans la parcelle claire soumise à une forte éclaircie où le feuillage moins abondant a été plus rapidement saturé.

S'il est évident que l'intensité du traitement appliqué détermine le niveau de l'égouttement, il reste à envisager pour la croissance secondaire de l'arbre, les conséquences d'une plus grande disponibilité en eau. En cette manière, ni le rôle fondamental de l'eau dans les processus de croissance, ni loccurrence en période chaude d'un déficit hydrique temporaire au niveau du sol ne restent à démontrer (Kozlowski \& Kramer, 1979). D'autre part, Kramer (1966) et Kozlowski (1955 et 1958) ont déjà souligné le lien existant entre le stress hydrique du substrat et celui qui, considéré comme un des facteurs régulateurs de la croissance, est enregistré au cour même de l'arbre.

Toute quantité d'eau supplémentaire qui atteint le sol en période de sécheresse relative durant la phase de croissance secondaire, contribue au maintien ou à l'amélioration du taux d'accroissement individuel en diamètre. Or, cette quantité est d'autant plus élevée que l'éclaircie est forte.

L'augmentation du taux de croissance mise en évidence dans les parcelles d'épicéas fortement éclaircies, trouve vraisemblablement dans l'amélioration du taux d'égouttement un facteur d'explication significatif.

\section{Summary}

Drip in young super-fir stands in relation with the intensity of the first thinning.

The intensity of first thinning in young stands of spruce ( $P$. abies Karst.) directly influences the current yield of these stands. This can be partially explained by a modification of the environment of the trees.

One of the components of the water balance - the drip - has bcen measured during the growing season in two stands of differently thinned $\left(16,5\right.$ and $\left.24 \mathrm{~m}^{2} / \mathrm{ha}\right)$ spruce trees. 
The results show that the drip rate has been increased by $8,4 \mathrm{p} .100$ in the more intensively thinned stand $\left(16,5 \mathrm{~m}^{2} / \mathrm{ha}\right)$ as compared to the other one $\left(24 \mathrm{~m}^{2} / \mathrm{ha}\right)$. In the former, the average minimum value of rainfall needed to have a drip was reduced by $27 \mathrm{p}$. 100. This higher proportion of water reaching the ground level is beneficial for the stand, especially in drought periods.

It is suggested that this could partially explain the observed higher growth rate of the stands intensily thinned.

Reçu le 14 juin 1983.

Accepté le 28 novembre 1983.

\section{Références bibliographiques}

ANdré P., 1976. Premic̀res éclaircies en peuplement résineux. Bull. Soc. R. F. Belg., 6, 312-331.

André P., Blerót P'h., 1978. Normes d'éclaircies en futaie équienne résineuse. Bull. S. R. F. Belg., 1, 1-19.

Aussenac G., 1968. Interception des précipitations par le couvert forestier. Ann. Sci. For., 25 (3), 135-156.

Aussenac G., 1969. Influence du couvert forestier sur les précipitations. Rev. For. Fr., 7, 631-635.

Aussenac G., Granier A., Naud R., 1982. Influence d'une éclaircie sur la croissance et le bilan hydrique d'un jeune peuplement de Douglas (Pseudotsuga menziesii (Mirb.) Franco). Can. J. For. Res., 12 (2), 222-231.

Brechtel, J., 1969. Deutsche Gewasserkindl., Mitt. Sonderheft, 24-31.

Bultot F., Dupriez G.L., Boudeux A., 1972. Interception de la pluie par la végétation forestière. Estimation de l'interception journalière à l'aide d'un modèle mathématique. J. Hydrol., 17 (3), 192-223.

DELFs J., 1967. Interception and stemflow in stand of Norway spruce and Beech in WestGermany, Int. Symp. of Forest Phytology, Pergamon Press, 179-185.

Kalma J.D., Stanhill G., Uriflli E., 1968. Rainfall interception and stemflow in one Orange plantation. Isr. J. Agric. Res., 18 (1), 3-14.

KozLowsKi T.T., 1955. Tree growth, action and interaction of soil and other factors. J. Forest., 53, 508-512.

Kozlowski T.T., 1958. Water relations and growth of trees. J. Forest., 56, 498-592.

Kozlowski T.T., Kramer P.J., 1979. Physiology of Woody Plants, Academic Press, 811 p.

Kramer P.J., 1966. Crown development in conifer stands in Scotland as influenced by initial spacing and subsequent thinning treatment. Forestry, 39 (1), 440-443.

LeONARD R.E., 1961. Net precipitation in a Northern Hardwood Forest. J. Geophys. Res., 66 (8), 2417-2421.

Mitscherlisch G., Moll W., 1970. Unterschungen über die Niederschlags- und Boden Feuchtigkeitsverhältnisse in einigen Nadel und Laubholzbeständen in der Nähe von Freiburg. Alg. Forst. u. J. Ztg., 141 (3), 49-60.

NoIRliduise A., 1959. Sur l'interception de la pluie par le couvert dans quelques forêts belges. Bull. Soc. R. For. Belg., 66 (10), 433-439.

ReYNolds E.R.G., Henderson C.S., 1967. Rainfall interception by Beech, Larch and Norway spruce. Forestry, 40 (2). 165-184.

WEIHE, 1968. Interception of rainfall by BeBen and Spruce. Allg. Forstzeitshr., 23 (6), $86-90$. 